\title{
Towards Business Ontologies Matching for Inter-Enterprise Collaboration Platform in a Lean Manufacturing Strategy
}

\author{
Ahlem Zayati ${ }^{1}$, Lilia Sidhom ${ }^{2}$, Youakim Badr ${ }^{3}$, Frédérique Biennier ${ }^{3}$, \\ and Mohamed Moalla ${ }^{4}$ \\ ${ }^{1}$ INSA de Lyon - Laboratoire LIESP - F69621 Villeurbanne Cédex, \\ France \& Faculté des Sciences de Tunis, Laboratoire LIP2 \\ ahlem.zayati@insa-lyon.fr \\ ${ }^{2}$ INSA de Lyon - Laboratoire AMPER - F69621 Villeurbanne Cédex, France \\ lilia.sidhomainsa-lyon.fr \\ ${ }^{3}$ INSA de Lyon - Laboratoire LIESP - F69621 Villeurbanne Cédex, France \\ \{frederique.biennier, youakim.badr\}@insa-lyon.fr \\ ${ }^{4}$ Faculté des Sciences de Tunis, Laboratoire LIP2, Tunisie \\ mohamed.moalla@fst.rnu.tn
}

\begin{abstract}
The Lean Manufacturing strategy aims at developing the enterprise value chain concept. The Lean makes extensive use of new organizational strategies such as business refocusing and development strategies for interenterprise collaboration. These reorganizations involve agility to deal with structural changes supported by communication technologies. However, the multiplicity of enterprise IT leads to complicate the inter-enterprise collaboration. However, the Services-Oriented Architecture provides flexibility and openness to enterprise Information System thanks to the "service" notion but fails to deal with business semantic mediation between partners involved in the collaboration. To meet these issues, this paper focuses on a semantic Enterprise Services Bus (ESB) which is a Service-Oriented Architecture middleware to define a business ontology-based matching and provide business and technological alignment to homogenize the information exchanged in the whole collaboration network. This paper also focuses on the technical implementation of the semantic ESB.
\end{abstract}

Keywords: Virtual Enterprise, Service Oriented Architecture (SOA), Enterprise Interoperability Business Ontologies \& Industrial Service.

\section{Introduction}

The economic context evolution leads to extending supply chains and inter-enterprise collaboration strategies. It fosters the development a global value chain which transcends beyond individual organizations to be applied to the entire supply chains and collaborative networks. The Lean Manufacturing strategy involves the extensive use of new organizational strategies in addition to the business refocusing strategy. It proceeds from the customer demands and defines the enterprise value, and then it 
streamlines processes, eradicates wastefulness and improves enterprise interoperability [13]. The inter-enterprise collaborations involve agility and call for a coherent Information System (IS). However, advances in enterprise technologies tend to increase and lead to complicate the inter-enterprise collaboration. However, legacy enterprise IS is not consistent and presents a lack of interoperability. In this context, the Service-Oriented Architecture (SOA) deals with technical inconsistencies and provides flexibility and openness through the "service" notion. However SOA turns out to be a solution for ensuring a technological interoperability and fails deal with business semantic mediation between all the parts of the collaboration.

To meet these issues, we introduce a collaborative platform in order to create extended and global value chains. Therefore we propose to adopt the alignment between enterprise IS and its business strategy to ensure flexibility and reactivity. We particularly pay attention to ensure interoperability within the collaboration network at different levels namely conceptual, organizational and technological.

The remaining of the paper is organized as follow: In section 2, we present the context and the state of the art. Section 3 introduces our contribution and section 4 depicts the conclusion.

\section{Context and State of the Art}

An efficient economic activity aims at increasing production output and relying on optimal consumption of services and goods. It tends to use available resources to maximize the production by taking into account the cost factor [13]. To meet this issue, collaboration strategies emerge to provide flexibility of resources usage and production efficiency. In addition, the Lean Manufacturing strategy aims to extend the enterprise value chain to a global value of virtual enterprise [13]. It involves the emergence of an inter-enterprise collaboration strategy which is based on collaborative processes composed as dynamic choreography of services according to customer requirements. As result, inter-enterprise collaboration urges open, agile and interoperable information system. Some works introduce perspectives of inter-enterprise collaboration as "4C-model" [5] which provides a collective business processes and activities for collaborative context considering the capacities of resources, the product cost and the validity of distributed information. However the basis of a Lean strategy is to eliminate wastefulness. That is why this approach is not appropriated to such production strategy because authors don't emphasize the monitoring and supervision of production processes.

In general, the enterprise IS has to deal with the following interoperability levels [3]:

- Business Level: refers to the global enterprise organization harmonisation

- Process Level: concerns processes interaction according to the enterprise specific needs.

- Service Level: identifies, composes and creates common functions for enterprise applications taking into account semantic and syntactic aspects.

- Data Level: refers to data models in a collaborative work and a conceptual schema organization. 
However, enterprise existent IS outlines some interoperability problems as outlined by [4]:

- Conceptual problem concerns semantic and syntactic presentation of high level information modelling.

- Technological problem is related to the inconsistency of information technologies.

- Organizational problem is associated to authority and responsibility definitions of work conditions.

To face the technological interoperability problem, the SOA implements IS components in a loosely coupled approach [7] in order to interconnect distributed IS components. The Enterprise Service Bus (ESB) is a SOA middleware which enables service integration and distributed communication services, and provides access to multiple heterogeneous applications [16]. The SOA can implement a collaborative platform but it fulfils only a syntactic mediation and improves technological interoperability. The ESB does not take into account semantic mediation and organizational interoperability. The ontology can deal with semantic mediation. It refers to the shared understanding of some domain of interest which may be used as a unifying framework to solve a semantic problem and provides a semantic interoperability when acting as a communication medium. The mediation part is used to process messages mechanism through the middleware and enables modifying, transforming, routing messages to be delivered according to some conditional logic in the mediation [6]. ARPA I3 architecture [11] can deal with semantic mediation. It distinguishes three interesting service classes among five: coordination services (discovery, invocation), semantic transformation services (ontology translation, process integration) and adaptation services (event managing, form conversion). ARPA I3 meets semantic mediation but does not emphasize business and monitoring services but does not consider organizational interoperability. To overcome this limit, one can consider the Enterprise Ontology (EO) and ISA S-95 to deal with organizational interoperability. First, the EO presents enterprise organization. Then, ISA S-95 standard depicts the product lifecycle when introducing production models and performance. It contains most of the general terms relating to an enterprise such as sales, activities, strategies and can serve as the basis for a shared understanding of an organization [12]. This ontology is conceptually divided into different sections as Meta-Ontology, Activities and Processes, Organization, Strategy and Marketing and presents a global view of the organization as well as an integration support which brings flexibility and coherent communication between different enterprise views [15]. ISA S-95 is the international standard for the integration of enterprise and control systems through the development of automated interfaces between enterprise and control systems [1] fitting mostly interfaces between enterprise and production control systems by specifying which information should be exchanged between ERP and MES systems. Therefore ISA S-95 can define pivot ontology. To overcome organizational interoperability problem, we purpose to take into account the Enterprise Ontology and ISA S-95 standard as pivot business ontologies. Therefore, we propose to develop ISA pivot ontology and then to match EO and ISA S-95. We mainly distinguish two matching mechanisms: 
- The elementary matching can be performed as a non-logical matching based on text similarity measurement or path-length based similarity of concepts and as logical matching based on deductive approach

- The hybrid matching is a combination of logical and non-logical matching mechanism [2].

The YASA4WSDL matchmaker supports multiple ontologies to annotate semantically services. It relies upon elementary matching with exploiting a logic-based approach. It extends SAWSDL, a basic type of annotation (the model reference and the schema mapping) for enhancing expressiveness of service description. As seen previously different works are done in this interoperability field but they lack of interpreting the different levels. Our goal is to couple the business and technological levels to set a "Lean" middleware to support enterprise interoperability.

\section{A Collaborative Platform Based on a Semantic ESB}

The inter-enterprise collaboration has to take into account both business and technological interoperability constraints and requires an alignment between enterprise technologies and its business strategy to ensure flexibility and reactivity. Thus implies that the industrial and technological contexts have to be integrated in the organisation to support organisational and technological interoperability in the same time. This involves adding semantic annotation to capture the business related to knowledge and integrating them with the technological level. To fit this goal, we propose a Lean ESB (see Fig. 1) extending the classical ESB middleware with business semantic management to support inter-business area mediation and business alignment [14].

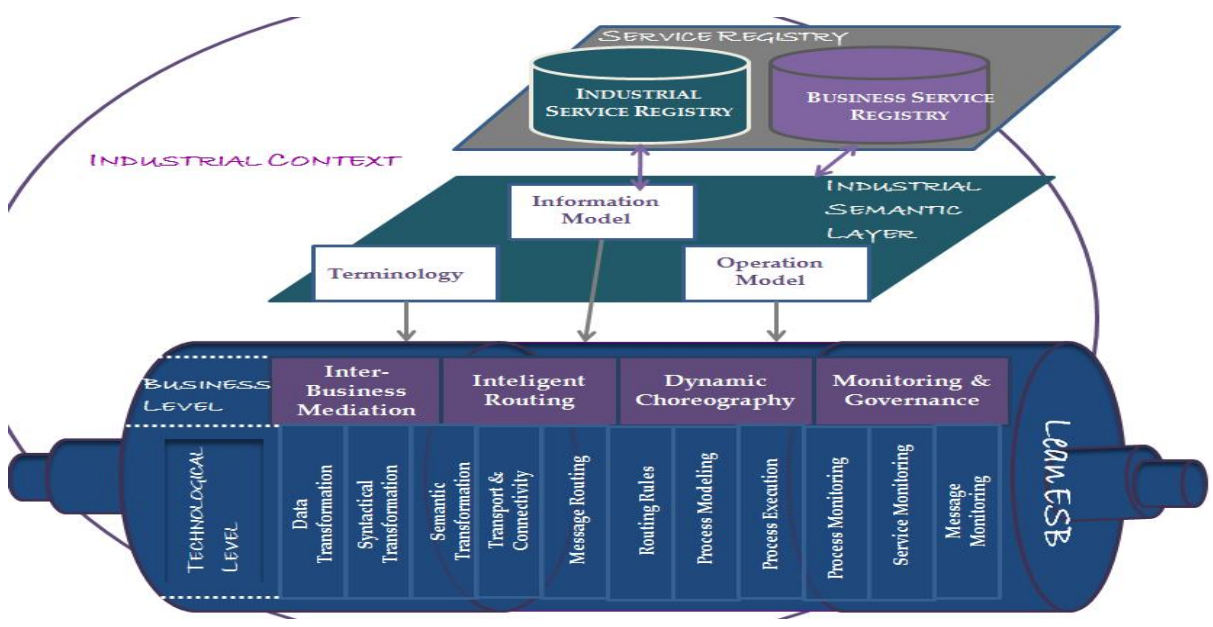

Fig. 1. The Collaborative Platform Global Organization

The semantic layer is related to business modules as well as industrial service registries [15]. The work presented here focuses on defining a single industrial registry which contains the matching of all industrial constraints such as production definition, organizational entities, physical processes, product definition, etc. 


\subsection{Business Semantic Integration}

The purposed Semantic ESB aims to define a single industrial services registry which contains the matching results of all industrial constraints such as production definitions, organizational entities, physical processes, product definitions etc Industrial services chains are organized through a selection process which takes into account functional industrial service properties (i.e. product, material constraints, rules management) and non-functional industrial service properties (i.e. quality constraints, security and process maturity). Constraints related to functional and non-functional properties are defined in terms of Service Level Agreements (SLA) [15]. The industrial service annotation refers to ISA S-95 semantics as illustrated in Fig 2.

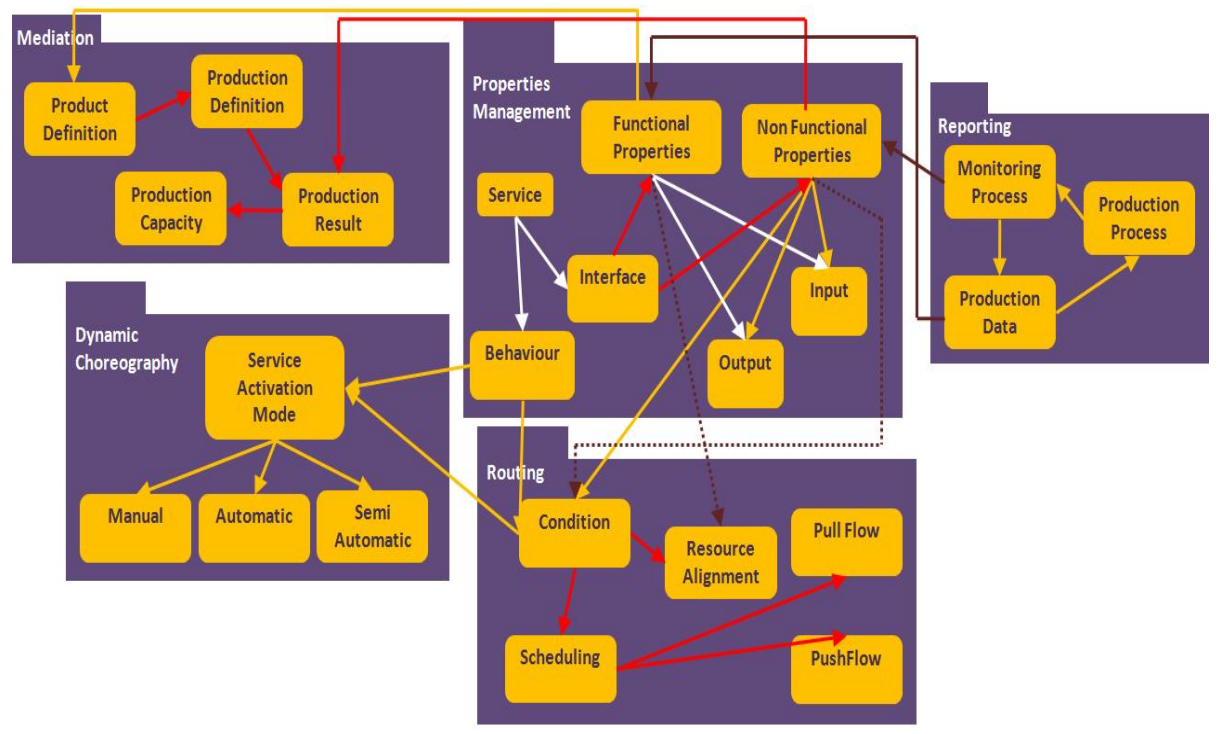

Fig. 2. The Industrial Service Model

In fact, the ISA S-95 standard enables the integration of production management and control systems (see Fig 3a, b, c) as it gathers different models (production definition, production schedule, production performance, production capacity and maintenance models).

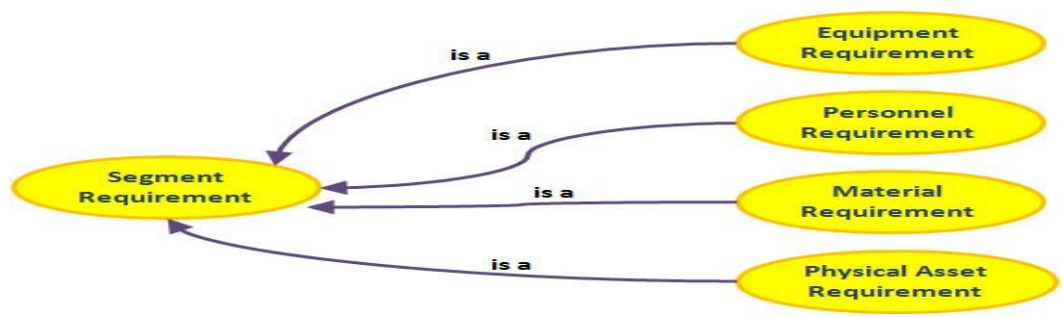

Fig. 3(a). The ISA S-95 Ontology - Production Tools 


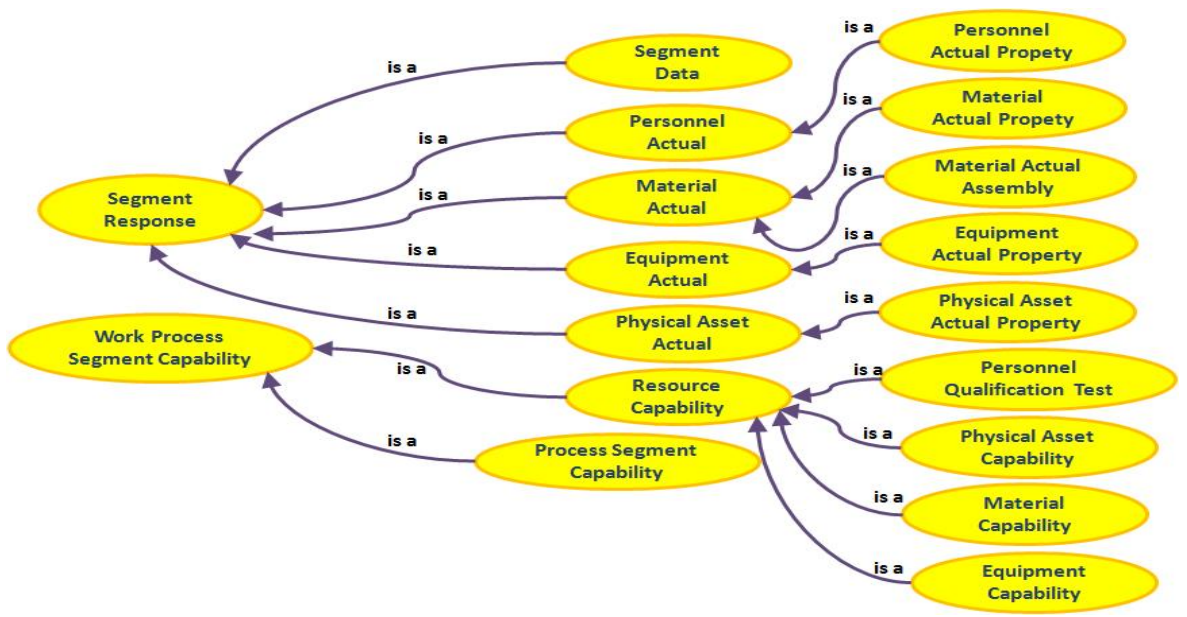

Fig. 3(b). The ISA S-95 Ontology - Resource Detail

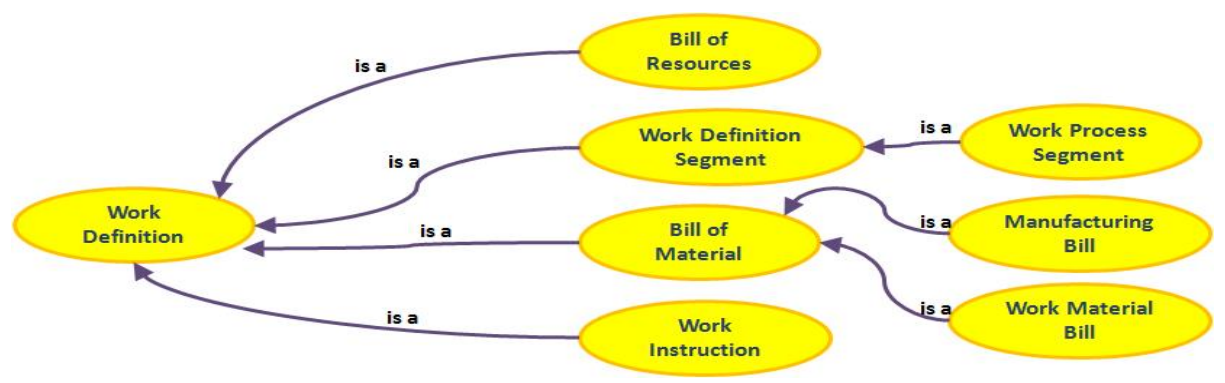

Fig. 3(c). The ISA S-95 Ontology - Operative scale

This is why we adopt it to develop the pivot ontology. Each industrial service description respecting ISA S-95 ontology is set as semantic annotations. As we have to mix technological and domain related annotations, we use YASA4WSDL to integrate these two kinds of annotations. The technological ontology refers to service semantics (input, output, operation, behaviour) whereas the domain ontology refers to the business interpretation of these data (Fig. 4). In the case of a Production Execution for example, we define industrial services according to the ISA S-95 ontology: When receiving a customer order, a message will be delivered to the workshop including product specifications, delivery delay, and requested quantities of finished products. It is defined as a "Work Request". Although, the ISA S-95 ontology provides a full definition of the production concepts, it does not meet the interoperability problems between the production management and organization and the production control. This is why the organizational ontology EO must be integrated. 


\subsection{Industrial Services Composition and Orchestration}

Collaboration strategy hinges on a set of collaborative processes. In a Lean Manufacturing strategy, collaborative processes are dynamically composed by services choreography according to customer demands. Therefore, semantically annotated services are selected to compose "just in time" processes. To fit inter-business area interoperability, the business choreography includes a routing part to set business matching thanks to our ISA S-95 ontology and then uses this transformed service in the service chain. The ISA and EO ontologies matching provide an end-to-end business and technological interoperability mixing service and business annotations (see Fig. 4).

According to our previous example, "Customer Order" and "Product Features" define production processes and production scheduling. The EO ontology defines "Features of Product" and "Strategic Plan" which assist production process definition and business strategy. The "Organizational Entities" define resources which take a part in business processes. Therefore, YASA4WSDL improves a schema-based mapping among the two business ontologies in order to define industrial services orchestration.

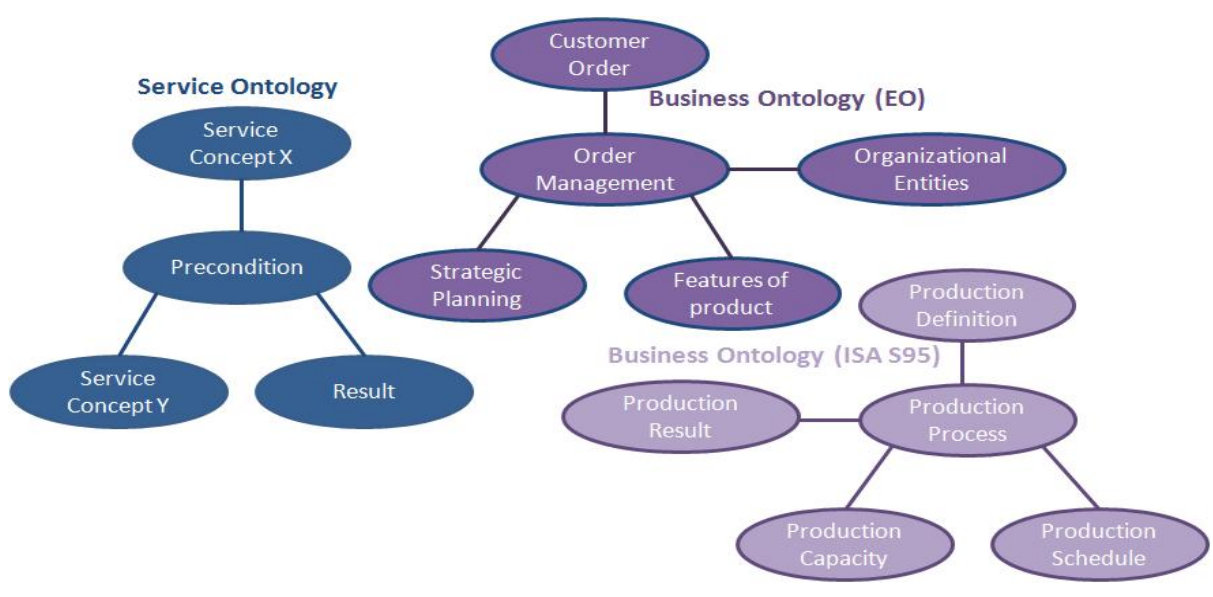

$<$ operation name = "customerOrderManagement"

serviceConcept $=$ "\&ServiceOntology\#precondition\&ServiceOntology\#result"

productionSchedule $=$ "\&BusinessOntology\#customerOrder\&BusinessOntology\#featuresofProd uct\&BusinessOntology\#ProductionDefinition \&BusinessOntology\#productionCapacity” organizationalEntities = “\&BusinessOntology\#orderManagement\&BusinessOntology\#featureof Production\&BusinessOntology\#productionProcess\&BusinessOntology\#productionSchedule” productionResult= "BusinessOntology\#customerOrder\&BusinessOntology\#managementOrder \&BusinessOntology\#productionDefinition”>

Fig. 4. Service Ontology and Domain Ontology (Business Pivot Ontology)

Lastly, this business choreography launches the industrial monitoring process when a service is triggered. This monitoring process governs the industrial service quality and concurrently monitors production processes. Then performance indicators are different from those saved in a regular situation, the monitoring process detects a 
production failure. In this case, the routing process tunes resource and service allocation so that production process will not fail.

\section{Conclusion}

In this paper, we proposed an industrial service middleware based on a semantic ESB infrastructure and on a dedicated industrial service registry. This middleware rests on a pivot business ontology (built from EO and ISA S-95 ontologies) to improve technological and business alignment. Industrial services are annotated according to different business ontologies before being "translated" in a pivot form (based on EO and ISA S95 ontologies) thanks to the YASA4WSDL framework.

Our prototype extends the SemEUsE semantic ESB [8] band uses the open source Dragon [9] framework to store and retrieve services with respect to their semantically defined functional and non-functional properties.

\section{Acknowledgement}

This work is supported partly by the ANR SemEUsE Project under grant 2007, TecLog 018 and by the Rhone-Alpes Area Council via the Cluster GOSPI SimSyProd project.

\section{References}

1. ANSI/ISA: Enterprise Control System Integration - Part I: Models and Terminology for Enterprise-Control System Integration (2007)

2. Chabeb, Y., Tata, S.: Yet Another Semantic Annotation for WSDL (YASA4WSDL). In: Proceedings of the IADIS WWW/Internet 2008 Conference, pp. 437-441 (2008)

3. Chen, D.: Enterprise Interoperability Framework. In: Workshop on Enterprise Modelling and Ontologies for Interoperability, EMOI-INTEROP (2006)

4. Daclin, N., et al.: Evaluation de l'Interopérabilité Organisationnelle et Managériale des Systèmes Industriels: le projet Carioner, CEROM - MTO (2008)

5. Ermilova, E., Afsarmanesh, H.: Competency Modelling Targeted on Promotion of Organizations towards VO Involvement. IFIP, vol. 283, pp. 3-14 (2008)

6. IBM WebSphere Developer Technical Journal: A practical introduction to message mediation - Part 1, http: / /www . ibm. com/developerworks / websphere/

7. Khoshafian, S.: Service Oriented Enterprise. Auerbach Publications, Taylor \& Francis Group (2007)

8. Petals White Paper, http://petals.ow2 org/docs/PEtALS-Architecture3-07-09.pdf

9. SEmEUse Project, http: //www. semeuse.org/architecture.html

10. Schmidt, M.T., Hutchinson, B., Lambros, P., Phippen, R.: The Enterprise Service Bus: Making Service Oriented Architecture Real. IBM System Journals, 781-797 (2005)

11. Touzi, J., Bénaben, F., Pingaud, H.: Model transformation of collaborative business process into mediation information system. In: IFAC World Congress 2008 (2008), http://tc.ifac-control.org/5/3/activities/tc5-3-sessions-atwC2008/c24-presentations/SeoulJTFBHP.pdf 
12. Uschold, M., Gruninger, M.: ONTOLOGIES: Principles, Methods and Applications. Knowledge Engineering Review 11(2) (1996)

13. Womack, J., Jones, D.: Système Lean, Penser l'entreprise au plus juste, 2nd edn. (2005)

14. Zayati, A., Sliman, L., Biennier, F., Badr, Y., Moalla, M.: Framework de Bus de Services de Data Mining pour un Système d'Information Industriel. In: SETIT 2009-IEEE (2009) ISBN 978-9973-0-0122-1

15. Zayati, A., Biennier, F., Badr, Y., Moalla, M.: Towards Lean Service Bus Architecture for Industrial Integration Infrastructure and Pull Manufacturing Strategies. Journal of Intelligent Manufacturing no. 10845, ISSN 0956-5515

16. Zhao, Y.Z., Zhang, J.B., Zhuang, L.Q., Zhang, D.H.: Service-Oriented Architecture and Technologies for Automating Integration of Manufacturing Systems and Services. In: ETFA 2005, pp. 349-355 (2005) 